\title{
MUI-Suppression with Lattice Structures for UTRA FDD Downlink
}

\author{
Klaus Knoche, Jürgen Rinas and Karl-Dirk Kammeyer \\ Department of Communications Engineering \\ University of Bremen \\ Otto-Hahn-Allee, D-28359 Bremen, Germany \\ Email: $\{$ knoche, rinas, kammeyer $\} @$ ant.uni-bremen.de
}

\author{
Wen $\mathrm{Xu}$ \\ Siemens AG - Mobile Phones \\ Grillparzerstr. 10 - 18 \\ D-81675 Munich, Germany \\ Email:Wen.Xu@siemens.com
}

\begin{abstract}
Wideband CDMA systems with orthogonal spreading codes suffer severely due to the loss of orthogonality by multipath propagation. This yields Multiple User Interference (MUI) which gravely reduces the performance of classical systems with Rake-receivers.

In our approach we attempt to restore orthogonality again by using a Lattice equaliser. These kind of equalisers are composed of two parts. The prewhitening part and the adaptive equaliser part. The Lattice structure itself has the advantage that its backward prediction errors are orthogonal to each other. Therefore the Lattice structure itself is working as a prewhitening filter. The second part is the adaptive algorithm for the equaliser coefficients. We take the stochastic gradient algorithm for this task and use the pilot channel in UMTS as a reference to calculate the equaliser coefficients. In order to show the feasibility of this approach, some simulation results for channels with low Doppler frequencies are presented and compared with the a system using the classical Rake-receiver and a system with another prefilter approach, the LMS-like Griffith equaliser [1].
\end{abstract}

\section{INTRODUCTION}

UMTS is the 3rd generation mobile cellular communication system in the $2 \mathrm{GHz}$ band. Upon others, it uses a W-CDMA scheme. One of the major problems of these kind of systems is the degradation of the performance due to Multi User Interference (MUI) which inherits from the multipath propagation of the mobile time variant channel. The classical Rake-receiver structure can not cope with it, even in a partly loaded system. Due to this, another solution for the receiver has to be found. Looking at the downlink on the other side, power consumption of the cellular is also a strong issue. Therefore algorithms must be simple in terms of computational effort. This reduces our set of possible solutions radically. Because the UMTS FDD downlink uses a long code for scrambling, approaches which take advantage of the the cyclostationary of short codes are of no use either. It was shown that at least major parts of the MUI can be modeled as coloured noise. This results in an approach to adapt the Rake-receiver to coloured noise [2]. Another feature of UMTS is the orthogonality of its spreading codes, the so called Orthogonal Variable Spreading Factor (OVSF) codes. Of course it gets lost on the way between base station and cellular due to the multipath propagation channel. Therefore another possibility to fight intra-cell MUI, is to re-establish this orthogonality of these codes by using a zero-forcing approach or try to find a compromise between

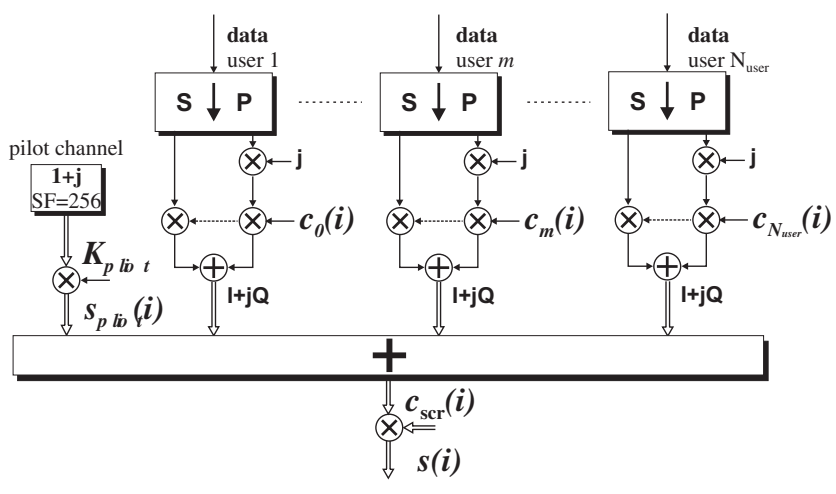

Fig. 1. Simplified transmitter scheme

noise enhancement and orthogonality with a Wiener scheme [3]. This shall be our approach too.

Since the direct approach is quite expensive in terms of computational effort many approaches use some iterative algorithm like RLS or LMS to adapt its coefficients to the time variant channel [4] [1]. The resulting systems look either like the classical rake receiver with an additional prefilter depicted in figure $2 \mathrm{a}$ ) or a prefilter with a correlation receiver as it is displayed in figure $2 b)^{1}$. A typical example of this scheme is the approach proposed in [1], which is taken as reference within this paper. In this paper we focus mostly on the application of a Lattice structure for a W-CDMA system itself and present a few simulation results, instead of its full derivation.

\section{SySTEM MODEL}

We have taken a CDMA-System and consider the whole system as a chip rate model. Upon others UMTS defines a FDD wide band CDMA scheme. In this paper only the downlink is considered. The spreading is done with the help of an OVSF code (Walsh) $c_{m}(i)$ with a spreading factor between 4 and 512 and an additional scrambling with a truncated complex gold code $c_{\mathrm{Scr}}(i)$ with the length of one frame equals 38400 chips or $10 \mathrm{~ms}$. Every frame consists of 15 slots with a length 2560 chips each. In our case the scrambling code is the same for every user in one cell. In general a QPSK modulation

\footnotetext{
${ }^{1}$ Each system can be transformed to the other one and vice versa.
} 


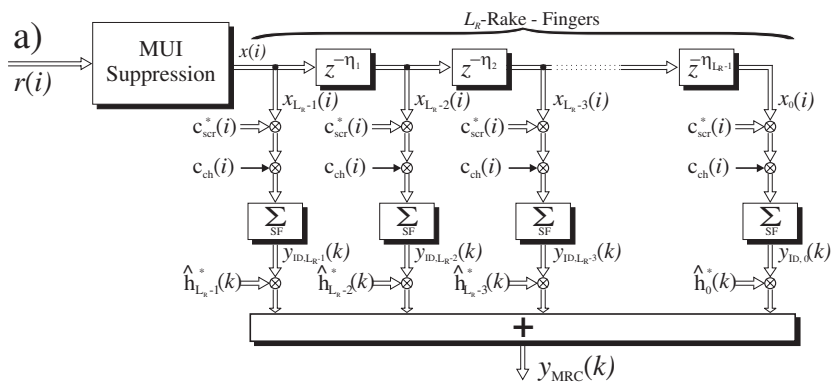

$I \& D$

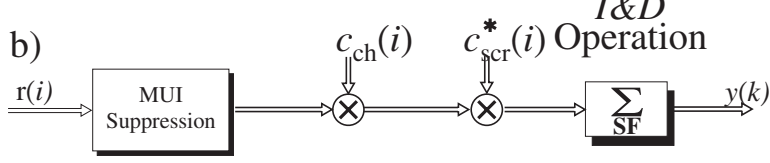

Fig. 2. MUI-schemes

is used in downlink direction ${ }^{2}$. The data $d_{n}(k)$ of the $\mathrm{n}$-th user with index $k$ denoting the symbol rate is mapped to chip rate by

$$
\tilde{d}_{n}(i)=\sum_{k}\left(d_{n}(k) \cdot \sum_{l=0}^{G_{S F}-1} \delta\left(i-l-k \cdot G_{S F}\right)\right)
$$

The index $i$ denotes the chip rate. After this the signal is spread with its user dependent OVSF-code denoted with $c_{n}(i)$. The resulting signal at the transmitter output of the base station is the sum of all users multiplied with with the scrambling code $c_{\mathrm{scr}}(i)$ plus an additional pilot channel $s_{\text {pilot }}(i)$. In our case we assume that all users have the same spreading factor $G_{S F}$ and the same power.

$$
s(i)=\left(\sum_{n=1}^{N_{\text {user }}}\left(d_{n}(i) \cdot c_{n}(i)\right)\right) \cdot c_{\mathrm{scr}}(i)+s_{\text {pilot }}(i)
$$

The pilot channel has a fixed spreading factor, contrary to the ones used for the data, of 256. Its OVSF-code is a simple repetition code $(256 \times 1)$. The power of the pilot channel is $10 \%$ of the total transmit power of the considered cell and is represented by the amplification factor $K_{\text {pilot }}$. This simplified transmitter in respect to the UMTS standard, is depicted in figure 1.

$$
s_{\text {pilot }}(i)=K_{\text {pilot }} \cdot c_{\mathrm{scr}}(i) \cdot \frac{1+j}{\sqrt{2}}
$$

The output signal of the transmitter is convoluted with the time varying channel impulse response $h(i, \rho)$ and distorted by an additive white zero-mean Gaussian noise.

$$
r(i)=s(i) * h(i, \rho)+n(i)
$$

If we use a normal FIR-Filter with its coefficients $\mathbf{w}^{H}$ for equalising the channel influence, the resulting output signal $x(i)$ of the equaliser according to figure $2 \mathrm{~b}$ ) is

$$
x(i)=\hat{s}(i)=\mathbf{w}^{H} \cdot \mathbf{r}(i) .
$$

\footnotetext{
${ }^{2}$ We omit the special cases like HSDPA.
}

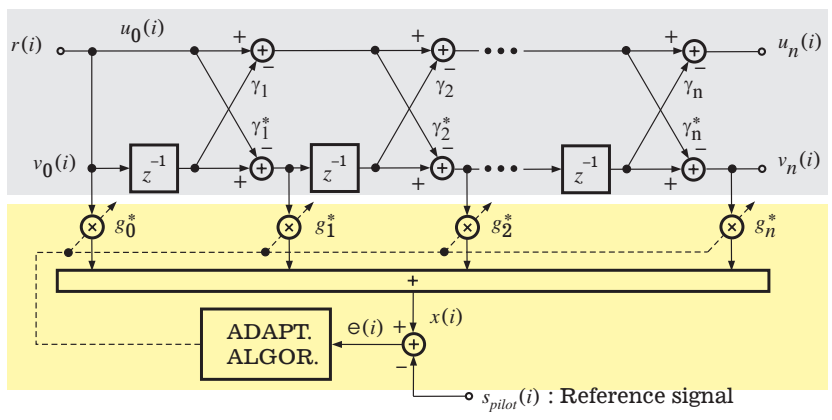

Fig. 3. Lattice equaliser

By correlating the signal $x(i)$ with the channelisation (OVSF) code and the scrambling code we receive an estimate for the data $\hat{d}(k)$ of the $m$-th user.

$$
y_{n}(k)=\hat{d}_{n}(k)=\sum_{i=k \cdot G_{S F}}^{(k+1) \cdot G_{S F}-1} x(i) \cdot c_{n}(i) \cdot c_{\mathrm{Scr}}^{*}(i)
$$

The question which arises, how to select the filter coefficients $w$. The classical solution for the coefficients $\mathbf{w}$ is solving the equation 7 .

$$
\mathbf{w}=\mathbf{R}^{-1} \mathbf{h}
$$

with $\mathbf{R}$ denotes the autocorrelation matrix and $\mathbf{h}$ is the vector with the channel coefficients.

As said before the direct solution is quite costly. Therefore an adaptive system for example LMS or RLS algorithm is used. Besides of the LMS or RLS algorithm we can also apply a Lattice (Ladder) structure to solve this problem.

\section{LATTICE EQUALISER}

In [2] the authors explain the statistical behaviour of MUI as coloured noise and therefore try to antagonise it with a Matched filter for coloured noise. Since this coloured noise seems to be a problem, the well known prewhitening capability of a Lattice structure can be used.

In figure 3 the Lattice equaliser is depicted. It consists of two parts, the Lattice structure itself and an equaliser part. The Lattice part can be derived from the linear prediction problem according to figure 4 .

We have to find the filter coefficient a which minimized the forward error $u$ The solution for this linear prediction filter is the Wiener Hopf equation.

$$
\mathbf{a}=\mathbf{R}^{-1} \phi_{\mathbf{r r}}
$$

With $\phi_{\mathbf{r r}}$ is the is the autocorrelation vector of the input signal $r(i)$. The autocorrelation matrix $\mathbf{R}$ has a hermitian Toeplitz structure. Due to this, it can be solved iteratively by the Levinson-Durbin recursion. Beginning with the linear prediction filter with order 0 , each prediction filter of higher order up to the wanted order is generated. Each of them has a forward prediction error denoted as $u_{m}$ for the $m$-th stage. Besides the filter a of order $m-1$ the so called PARCOR 


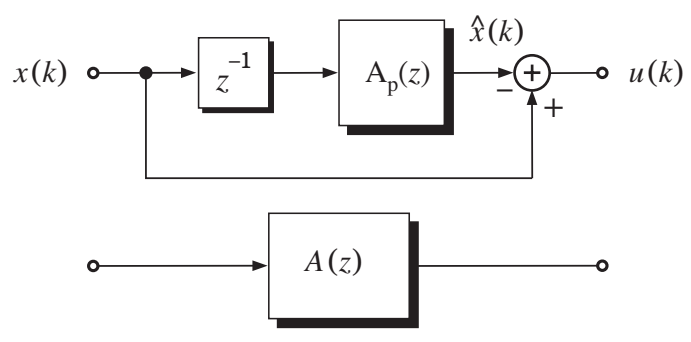

Fig. 4. Linear prediction filter

(PARtial CORrelation) coefficient $\gamma_{m}$ has to be known to calculate the next stage. It can be interpreted as the normalised partial correlation between the forward prediction error and $m$ times delayed input signal of the prediction filter $(x(i-m))$. Instead of calculating the filter coefficients a directly, it can be shown that a filter structure only consisting of the PARCOR coefficients $\gamma$ the forward prediction errors $\mathbf{u}$ and the backward prediction errors $\mathbf{v}$ is possible, the Lattice structure. The backward prediction error $v_{m}$ can be seen as error between a value $r(i-m)$ and its prediction from future values up to $r(0)$.

The connection between forward and backward prediction error $u$ and $v$ of the $\mathrm{m}$-th stage is displayed in equation 9 . An extensive derivation of the following properties can be found in nearly any good textbook concerning digital signal processing for example [5], [6], [7] or [8].

$$
\begin{aligned}
& u_{m}(i)=u_{m-1}(i)-\gamma_{m}(i) \cdot v_{m-1}(i-1) \\
& v_{m}(i)=v_{m-1}(i-1)-\gamma_{m}^{*}(i) \cdot u_{m-1}(i)
\end{aligned}
$$

In case of time invariant channel the PARCOR coefficient of the $m$-th stage $\gamma_{m}(i)$ is constant but in general $\gamma$ has to adapt to the change of the channel. It can be shown after a little algebra from [5] that an estimate of $\gamma_{m}(i)$ for a time variant channel can be obtained recursively by

$$
\begin{aligned}
\hat{\gamma}_{m+1}(i+1) & =\hat{\gamma}_{m+1}(i)+\frac{1}{b_{m}(i)} \cdot\left[u_{m}(i) v_{m+1}^{*}(i)\right. \\
& \left.+u_{m+1}(i) v_{m}^{*}(i-1)\right]
\end{aligned}
$$

and the shortcut $b_{m}(i)$ is also recursively defined as

$$
b_{m}(i)=\kappa \cdot b_{m}(i-1)+\left|u_{m}(i)\right|^{2}+\left|v_{m}(i-1)\right|^{2} .
$$

The forgetting factor $\kappa$ has to be chosen as a compromise between noise and time variance of the channel and is very close to one. Applying it to the UMTS-CDMA System it lies in the range between 0.999 and 0.9999 .

The power of each stage $m$ can also be calculated recursively by

$$
\sigma_{m}^{2}(i)=\kappa \cdot \sigma_{m}^{2}(i-1)+\left|v_{m}(i)\right|^{2} .
$$

In order to calculate the coefficients $g$ we first define the difference between the reference signal delayed by $i_{0}$ and the ongoing Lattice output. As reference we take the known pilot channel $s_{\text {pilot }}\left(i-i_{0}\right)$.

$$
\begin{aligned}
\epsilon_{m}(i) & =\left(\sum_{\nu=0}^{m} \hat{g}_{\nu}(i-1) \cdot v_{\nu}(i)\right)-s_{\text {pilot }}\left(i-i_{0}\right) \\
& =\epsilon_{m-1}(i)+\hat{g}_{m}(i-1) \cdot v_{m}(i) .
\end{aligned}
$$

The optimal delay $i_{0}$ has to be chosen according to the channel. For a minimum phase channel it has to be near zero, for a maximum phase system it should be set according to the order of the filter structure. This is also valid for the delay in LMS/RLS based schemes.

Using equation 13 according to [7] respectively [9] the new estimation of coefficients $\mathbf{g}(i)$ can be calculated by

$$
\hat{g}_{m}(i)=\hat{g}_{m}(i-1)-\frac{1}{\hat{\sigma}_{m}(i)^{2}} \cdot \epsilon_{m}(i) \cdot v_{m}^{*}(i) .
$$

\section{Simulation Results}

In the following we present some simulation results. Both simulations use a 4-tap Rayleigh fading channel. The system is always a little above a half loaded system ${ }^{3}$. This is quite relevant because cellulars should be capable to work in this environment. Besides of the proposed Lattice structure we take the classical rake receiver and the MUI-suppression scheme proposed in [1] and denoted with GLMS as second reference. In [1] the authors presented an adaptive solution for equation 7 by using a modified LMS-algorithm. Modified mainly in that way that instead of taking a reference the algorithm needs the channel impulse response $h(i)$ also known as Griffith's algorithm [10]. While the Lattice structure does its channel estimation (CE) indirectly for itself it has to be calculated for the Rake and GLMS. In our simulations we present two versions of it. First we assume perfect knowledge of the CIR. Second we estimate the CIR by correlating the received signal with the know pilot channel and averaging the results over one slot afterward. For every slot there is only one estimate for all coefficients of the CIR. A MA-filtering would be better of course and using a variable length MA-filter according to the velocity or another Wiener filter for $\mathrm{CE}$ would be even more beneficial. So these CE schemes would result in something in between perfect knowledge and our second assumed version. A channel coding was not applied. Additional references are the AWGN curve for QPSK modulated signals and the one user Rake-receiver curve for the taken environment. The order of both equalisers was set to 18 and the delay was set to 9 chips because we do not know about minimum or maximum phase and take a compromise. The higher the order of the equaliser gets as better the results are, but the performance of it in respect to the order has to be investigated thoroughly in the future.

In figure 5 the velocity is set to $3 \mathrm{~km} / \mathrm{h}$ and the spreading factor is 128 . The number of users is 64 plus the additional pilot channel which inherits $10 \%$ of the total power. The forgetting factor $\kappa$ is set to 0.9999 and the step factor for the GLMS equals $\mu=0.001$. This are not the optimal values because they change according to the noise. Nevertheless

\footnotetext{
${ }^{3}$ Due to the pilot channel
} 


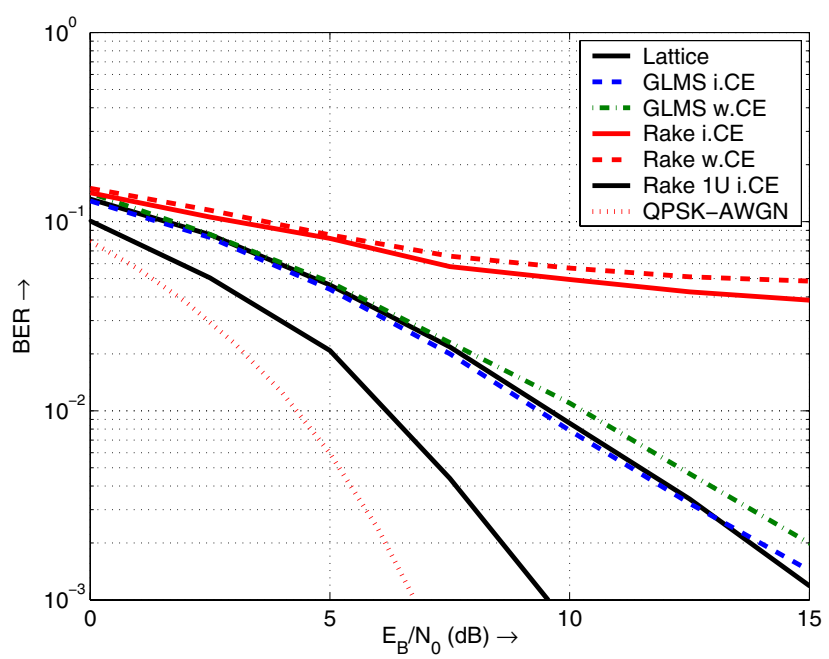

Fig. 5. Simulation results for 4 tap Rayleigh channel and $S F=128,3 \mathrm{~km} / \mathrm{h}$

they are near the optimal value at an $E_{b} / N_{0}=10 \mathrm{~dB}$. It can be easily seen that the Rake-receiver itself is not a very good algorithm in the presence of numerous users. The Lattice scheme is only slightly worse with approximately a quarter $\mathrm{dB}$ at a BER of $10^{-2}$ than the GLMS with perfect channel knowledge. The GLMS with CE is nearly as good as the Lattice in a noisy environment but degrades with higher $E_{b} / N_{0}$. At a BER of $10^{-2}$ it has a loss of approx. $0.7 \mathrm{~dB}$ compared to the Lattice and 0.9 to itself with ideal CE. At 15 $\mathrm{dB}$ the Lattice outperforms the GLMS with perfect channel knowledge but this is actually of no major interest because it is far beyond a possible working point.

In figure 6 we have taken the same scenario besides another spreading factor of 16 and the number of users is 8 . Because the simulations are made in respect to the $E_{b} / N_{0}$ of the transmitted data, the pilot channel's $E_{b} / N_{0}$ is 8 times higher resulting in a much better CE. This can also be seen in the simulation. Even the results of both Rake receivers are quite similar. The curves for the MUI-suppression schemes are now much closer to each other but the ranking is still the same. At a BER of $10^{-2}$ the Lattice has a loss of $0.15 \mathrm{~dB}$ compared to the GLMS with perfect CE and outperforms the GLMS with CE with about $1.5 \mathrm{~dB}$.

In [9] and others the adaptation speed of the Lattice equaliser was presented. It was shown that it adapts much faster than the LMS but worse compared to the RLS which needs much higher computational effort. But the major difference compared to [9] is the total different noise environment. On the other hand the performance of RLS/LMS equaliser schemes degrades fast in a high mobile environment [4]. The performance of the Lattice algorithm in a faster mobile environment has to be determined in the future.

\section{CONCLUSION}

In this paper a Lattice structure for a W-CDMA system for MUI-suppression was presented. It has a good performance in a pedestrian or nearly static environment but needs to be

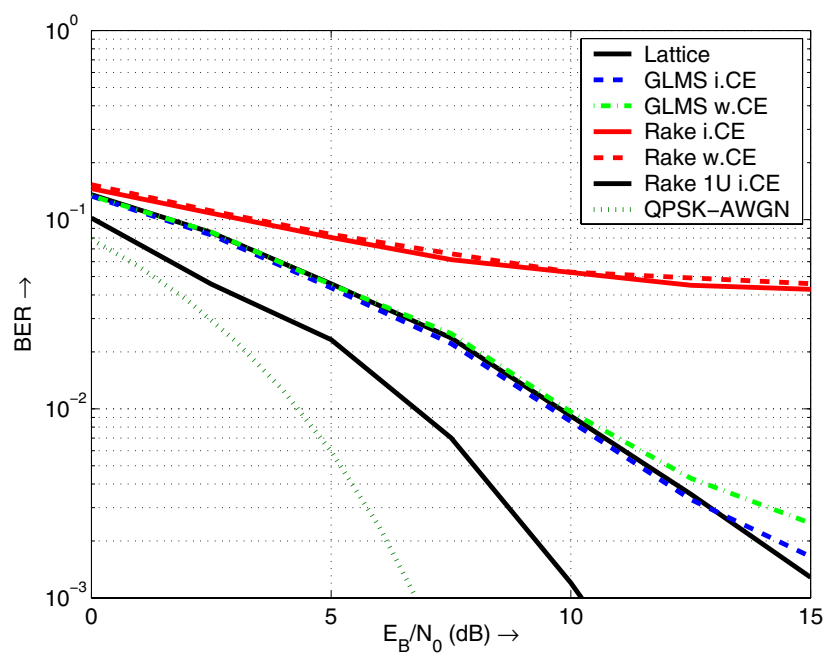

Fig. 6. Simulation results for 4 tap Rayleigh channel and $\mathrm{SF}=16,3 \mathrm{~km} / \mathrm{h}$

further examined for example in a high mobile environment. Other channel models have to be considered and the order of the equalisers compared to their performance gain have to be looked onto. In the presented approach the pilot channel was taken as reference. It may also be possible to apply the Griffiths approach [10] and take the value of the crosscorrelation between backward prediction error and pilot data instead of applying the pilot channel directly. After this crosscorrelation could be estimated and filtered instead of using a first order IIR filter like it was done here. This paper give a glimpse that the Lattice approach for W-CDMA works under special conditions but it needs to be further investigated.

\section{REFERENCES}

[1] M. J. Heikkilä, "A Novel Blind Adaptive Algorithm for Channel Equalization in WCDMA downlink," in Proc. IEEE Int. Symposium on Personal, Indoor and Mobile Radio Commun. (PIMRC), 2001.

[2] G. Bottomley, T. Ottosson, and Y.-P. E. Wang, "A Generalized RAKE Receiver for Interference Surpression," IEEE Journal on Selected Areas in Communications, vol. 18, no. 9, pp. 1536-1545, August 2000.

[3] C. Frank, E. Visotsky, and U. Madhow, "Adaptive Interference Suppression for Downlink of a Direct Sequence CDMA System with Long Spreading Sequences," 1998.

[4] O. Prätor, C. Unger, A. Zoch, and G. Fettweis, "Performance of Adaptive Chip Equalization for the WCDMA Downlink in Fast Changing Environments," in Proc. IEEE Int. Symposium on Spread Spectrum Techniques and Applications (ISSSTA), 2002.

[5] K. D. Kammeyer, Nachrichtenübertragung, 2nd ed. Stuttgart: Teubner, 1996.

[6] S. Haykin, Adaptive Filter Theory, 3rd ed. London: Prentice Hall International, 1996.

[7] J. G. Proakis, C. M. Rader, F. Ling, and C. L. Nikias, Advanced Digital Signal Processing, 1st ed. New York: Macmillan Publishing Company, 1992.

[8] J. G. Proakis, Digital Communications, 3rd ed. McGraw-Hill, 1995.

[9] E. H. Satorius and J. D. Pack, "Application of Least Squares Lattice Algorithms to Adaptive Equalization," IEEE Trans. on Communications, vol. 29, no. 2, pp. 136-142, February 1981.

[10] J. Treichler, C. J. Jr., and M. Larimore, Theory and Design of Adaptive Filters. New York, Chichester, Brisbane, Toronto, Songapore: John Willey and Sons, 1987.

[11] K. D. Kammeyer and K. Kroschel, Digitale Signalverarbeitung: Filterung und Spektralanalyse mit MATLAB Übungen, 4th ed. Stuttgart: Teubner, 1998. 